\title{
VALIDAÇÃo bRASILEIRA dO AlCOHOL CRAVING QUESTIONNAIRE - SHORT FORM REVISED (ACQ-SFR)
}

\section{BRAZILIAN VALIDATION OF ALCOHOL CRAVING QUESTIONNAIRE - SHORT FORM REVISED (ACQ-SFR)}

\author{
Renata Brasil Araujo ${ }^{1}$, Vinicius Jobim Fischer ${ }^{2}$, Daniel Capelli Fulginiti ${ }^{3}$, \\ Dan Roger Pozza ${ }^{4}$, Tharlise Paim Girelli ${ }^{3}$, \\ Nathália Sandoval Barbosa Mambrini ${ }^{3}$
}

\section{RESUMO}

Introdução: A avaliação do craving é importante para o tratamento de pacientes com Transtornos por uso de substâncias psicoativas. O objetivo deste estudo foi realizar a validação do Alcohol Craving Questionnaire-Short Form Revised- Versão Brasileira (ACQ-SFR - Versão Brasileira).

Métodos: Foi um estudo observacional transversal, com uma amostra não aleatória de 121 participantes alcoolistas, do sexo masculino, internados para desintoxicação em unidade especializada. Os instrumentos utilizados foram: entrevista clínica com dados sociodemográficos e de padrão de uso de substâncias, MMSE, ACQ-SFR - Versão Brasileira, EAV do craving, BDI, BAI, estímulo visual indutor de craving.

Resultados: $\mathrm{Na}$ análise fatorial, foram encontrados dois fatores: o fator 1 representa o craving pelo reforço positivo e negativo do álcool, e o fator 2 , o propósito de consumir o álcool associado ao prazer. O alfa de Cronbach total foi de 0,91 (12 itens), o do Fator 1 foi 0,92 ( 8 itens) e o do Fator 2 foi 0,70 (3 itens). $O$ total de pontos da escala foi correlacionado com a Escala Analógico-Visual $(r=0,658 ; p<0,01)$.

Conclusão: Conclui-se que o ACQ-SFR - Versão Brasileira demonstrou ter propriedades psicométricas satisfatórias.

Palavras-chave: Craving; validação; álcool

\section{ABSTRACT}

Introduction: The assessment of craving is important in the treatment of patients with psychoactive substance use disorders. The purpose of this study was to validate the Brazilian version of the Alcohol Craving Questionnaire - Short Form Revised (ACQ-SFR - Brazilian Version).

Methods: A cross-sectional observational study was conducted, with a non-random sample of 121 male alcoholic participants admitted for detoxification in a specialized unit. The instruments used to collect data were clinical interview with sociodemographic data and substance use pattern, MMSE, ACQ-SFR - Brazilian version, VAS of craving, $\mathrm{BDI}, \mathrm{BAI}$, and visual inducer of craving.

Results: In a factorial analysis, two factors were found: factor 1 represents craving due to positive and negative reinforcement of alcohol use, and factor 2 , the purpose of consuming alcohol associated with pleasure. Overall Cronbach's alpha was 0.91 (12 items), while factor 1 was 0.92 ( 8 items) and factor 2 was 0.70 ( 3 items). Total scale score correlated with VAS score $(r=0.658 ; p<0.01)$.

Conclusions: AACQ-SFR - Brazilian version presented satisfactory psychometric properties.

Keywords: Craving; validation; alcohol
Clin Biomed Res. 2019;39(1):15-23

1 Hospital Psiquiátrico São Pedro. Porto Alegre, RS, Brasil.

2 Institute for Health and Behaviour, University of Luxembourg,

Esch-sur-Alzette, Luxembourg.

3 Programa de Pós-graduação em Psicologia, Pontifícia Universidade Católica do Rio Grande do Sul (PUCRS). Porto Alegre, RS, Brasil.

4 Núcleo de Estudos e Atendimentos em Psicoterapias Cognitivas. Pontifícia Universidade Católica do Rio Grande do Sul (PUCRS). Porto Alegre, RS, Brasil.

Autor correspondente: Renata Brasil Araujo renataudbrasil@terra.com.br Hospital Psiquiátrico São Pedro, Unidade de Dependência Química. Clínica Modus Cognitivo,

Rua Mariante, 284, sala 501. 90430-180, Porto Alegre, RS, Brasil. 
O craving pode ser entendido, em sua forma mais básica, como um desejo ou vontade muito forte por algo ${ }^{1,2}$. Sua presença pode ser associada a uma série de situações e comportamentos do dia a dia como: uso de substâncias, consumo de alimentos, realização de sexo e participação em jogos eletrônicos ${ }^{3}$. Na avaliação do Transtorno por uso de substâncias psicoativas, a presença do craving é um dos critérios analisados nos dois manuais estatísticos de diagnósticos de doenças utilizados atualmente: a CID-10 e o DSM-5, ${ }^{4,5}$.

O craving não pode ser totalmente evitado em função de seu caráter imprevisível e automático apesar de terem situações nas quais seu risco é maior- e não saber lidar com ele faz dele um dos principais motivos de desistência dos tratamentos para dependência química, assim como da ocorrência de lapsos e recaídas ${ }^{6}$. Ele é influenciado pelo ambiente, em termos de gatilhos que podem desencadeá-lo, e por sentimentos negativos e de mal-estar, como ansiedade, tristeza, medo e estresse ${ }^{7,8}$.

São identificados quatro tipos de craving, cada um representando seus próprios motivos para o uso de substâncias psicoativas ${ }^{7}$. O primeiro seria como resposta à síndrome de abstinência, trazendo a vontade de usar novamente a substância de sua preferência para evitar o desconforto da privação; o segundo, como resposta à falta de prazer, quando buscam o uso para melhorar seu humor ou aumentar sensações de felicidade; o terceiro, como resposta condicionada a estímulos relacionados às substâncias psicoativas, que ocorre ao encontrar gatilhos como pessoas, locais, situações, horários, tipos de humor ou pensamento, os quais foram associados ao uso de substâncias; e, por fim, como tentativa de intensificar o prazer de determinadas atividades. Nesta última, o usuário relaciona uma substância ao melhor aproveitamento de uma determinada experiência. Exemplos podem ser o uso para melhorar a interação social, para maior aproveitamento e energia em festas e para maior potência e prazer sexual ${ }^{7}$. Devido à importância deste constructo, é fundamental que o mesmo seja avaliado, na clínica, e que escalas para sua mensuração sejam pesquisadas e validadas ${ }^{1,8-11}$.

Um instrumento desenvolvido para a avaliar o craving, é o Alcohol Craving Questionnaire (ACQ-NOW). O ACQ-NOW foi desenvolvido por Singleton, Tiffany e Henningfield ${ }^{8}$ e é composto por 47 itens, que são distribuídos em cinco dimensões: impulso, desejo de usar o álcool, intenção de usar o álcool, antecipação dos efeitos positivos, alívio do afeto negativo, e falta de controle sobre o uso. Cada dimensão do instrumento, é composta por 9 itens, do total de 47 , incluindo dois itens adicionais para checagem de consistência ${ }^{9}$.
Posteriormente, foi desenvolvida uma versão reduzida do ACQ-NOW8, o Alcohol Craving Questionnaire Short-Form Revised (ACQ-SFR) ${ }^{12,13}$, o qual possui 12, dos 47 itens da escala original. Esta versão reduzida foi desenvolvida para avaliar o craving pelo álcool,e distribuída em quatro fatores: compulsividade, expectativa, propósito e fatores emocionais. No que se refere à sua mensuração, manteve-se a avaliação pela escala Likert do ACQ-NOW ${ }^{8}$, que variava de Discordo Fortemente (1) a Concordo Fortemente (7). No teste de fidedignidade, os quatro fatores que abarcavam todos os 12 itens, foram fortemente correlacionados, sendo que o fator compulsividade, obteve um escore de fidedignidade de 0,79 , o fator expectativa e o fator propósito obtiveram um escore de 0,77 , enquanto os fatores emocionais obtiveram um score de $0,86^{12}$. Outros estudos realizados com o ACQ-SFR ${ }^{14,15}$, ao contrário da validação original, verificaram que o fator expectativas positivas tinham melhor consistência interna. O objetivo deste estudo é realizar a validação psicométrica e transcultural do ACQ-SFR ${ }^{12,13}$ para o Brasil, à medida em que se verifica a dificuldade e a importância de avaliar o constructo craving.

\section{MÉTODO}

Este foi um estudo observacional transversal. Para a validação de escalas nas quais é necessária a realização de uma análise fatorial, é preconizado que o tamanho ideal da amostra deve ser de 10 sujeitos para cada variável ${ }^{16}$, assim foram pesquisados 121 participantes, já que o ACQ-SFR tem 12 questões. A amostra foi por conveniência e composta por indivíduos adultos e do sexo masculino, que foram internados na Unidade de Desintoxicação do Hospital Psiquiátrico São Pedro, localizado na cidade de Porto Alegre, no estado do Rio Grande do Sul/ Brasil. Esse serviço interna, de forma voluntária, involuntária ou compulsória, pacientes com Transtornos mentais e comportamentais devidos ao uso de substâncias (com ou sem comorbidades psiquiátricas) pelo Sistema Único de Saúde (SUS), por uma média de 21 dias.

Eram critérios de inclusão ter o diagnóstico de Transtornos mentais e comportamentais devidos ao uso de álcool - síndrome de dependência pela CID-104 , estar utilizando esta substância há, pelo menos, um ano, ter um grau mínimo de escolaridade de quinta série do Ensino Fundamental e ter entre 18 e 65 anos de idade. Os participantes deviam estar abstinentes de substâncias entre 7 e 15 dias. Essas informações eram obtidas através de uma entrevista semi-estruturada com o paciente, na qual era preenchida uma ficha com dados sócio-demográficos 
e referentes ao padrão de consumo de substâncias psicoativas.

Apresentar sintomas psicóticos, estar em quadro agudo de algum Transtorno de Humor ou ter prejuízos cognitivos que alterassem o desempenho nos testes (avaliado pelo Mini-exame do Estado Mental ${ }^{17}$ ) eram fatores de exclusão.

\section{Instrumentos}

\section{Os seguintes instrumentos foram utilizados:}

1) Ficha com dados sócio-demográficos e referentes ao padrão de consumo de substâncias psicoativas - aplicada em entrevista semi-estruturada - com o objetivo de definir o perfil sócio-demográfico da amostra estudada, identificando características que podiam ser importantes para melhor avaliar se o indivíduo deveria ou não ser incluído no estudo, bem como seu consumo de substâncias psicoativas. Nesta também era questionada a quantidade de álcool consumida diariamente, sendo utilizada a medida "Unidades Internacionais de Álcool" - UI ${ }^{18}$.

2) Mini-exame do Estado Mental - Mini-Mental State Examination: $M M S E^{17}$ é um teste que tem como objetivo avaliar as condições cognitivas do paciente. Foi utilizado, nesta pesquisa, para excluir os pacientes com prejuízo cognitivo ${ }^{17}$.

3) Inventário Beck de Ansiedade ${ }^{19}$ - trata-se de uma escala sintomática, destinada a medir a gravidade dos sintomas de ansiedade. É composta por 21 itens em que o sujeito deve pontuar conforme os sintomas o afetam, em uma escala de quatro pontos. O escore total é obtido pelo somatório dos escores de cada item. Os pontos de corte para pacientes psiquiátricos foram conforme as normas da versão em português publicada em 2001 por $\operatorname{Cunha}^{20}$ (de 0 a $10=$ mínimo, 11 a $19=$ leve, de 20 a $30=$ moderado e de 31 a 63 = grave).

4) Inventário Beck de Depressão ${ }^{21}$ - é destinado a medir a intensidade dos sintomas de depressão, sendo formado por 21 itens, cada um com quatro alternativas. $O$ escore total resulta da soma dos pontos. Os pontos de corte para pacientes psiquiátricos foram publicadas em 2001, com a versão em português, também por Cunha $^{20}$ (de 0 a 11 = mínimo, 12 a 19 = leve, de 20 a 35= moderado e de 36 a 63 = grave).

5) Escala Analógico-Visual para avaliar o craving ${ }^{22}$ - o indivíduo era solicitado a dar uma nota para o seu craving, sendo 0 (zero) a nota mínima (não apresenta craving) e 10 a máxima (apresenta craving muito forte), marcando este valor em uma escala de 10 centímetros.

6) Alcohol Craving Questionnaire-SFR ${ }^{12,13}$ é uma escala abreviada da ACQ-NOW ${ }^{12}$ para avaliar o craving do álcool, sendo composta por 12 questões, diante das quais o indivíduo se posiciona utilizando uma escala likert de 7 pontos que vai de "discordo totalmente" até "concordo totalmente". Na sua elaboração, foi distribuída em 4 fatores denominados: Compulsividade, Expectativa, Propósito e Fatores emocionais. A versão brasileira, a partir de sua validação psicométrica, distribuiu-se em dois fatores: o fator 1 representa o craving pelo reforço positivo e negativo do álcool, e o fator 2 , o propósito de consumir o álcool associado ao prazer. A escala pode ser avaliada a partir de seu escore total (com as questões 3, 8 e 11 invertidas, devendo ser somadas às demais), a partir dos pontos do fator 1 (soma de todas as questões, exceto a $3,4,8$ e a 11) e do fator 2 (soma das questões 3,8 e 11 invertidas). A questão 4 não fez parte de nenhum dos dois fatores na validação brasileira. Os pontos de corte do total de pontos da escala foram: $12-17=$ craving mínimo, $18-25=$ leve, $26-42=$ moderado e 43 ou mais $=$ grave.

7) Fotos (duas), de tamanho $10 \mathrm{~cm} \times 14,8 \mathrm{~cm}$, para induzir o craving pelo álcool, sendo uma delas com a figura de um copo de cerveja e outra, com um copo de cachaça.

\section{Validação Semântica do ACQ-SFR}

Como a variável principal desta pesquisa é o craving, em um primeiro momento, após a autorização dos autores das versões em Inglês das escalas, foi realizado o Estudo Piloto I, para que o ACQ-SFR ${ }^{12,15}$ pudesse ser utilizado, sendo, este piloto, norteado por estudos anteriores ${ }^{22,23}$. Foram seguidos alguns passos:

1. Tradução do instrumento da Língua Inglesa para a Língua Portuguesa realizada por uma Professora de Inglês, graduada em Letras, habilitada na Língua Inglesa e conhecedora do objetivo da tradução.

2. Aplicação do instrumento traduzido em 10 sujeitos com a finalidade de verificar se as questões eram facilmente compreendidas e levantar possíveis dúvidas que poderiam ser suscitadas.

3. Brainstorming: Foram reunidos 5 sujeitos e cada item que compõe o instrumentos foi reproduzido verbalmente por eles, sendo questionado, neste 
momento, se o significado de cada questão estava claro.

4. Back-translation: A primeira tradução do instrumento foi revertida para o idioma de origem (Inglês) por um nativo de Língua Inglesa, com fluência na Língua Portuguesa e desconhecedor do objetivo da tradução.

5. Tradução do Instrumento: a partir do back-translation, o questionário foi novamente traduzido para a Língua Portuguesa por uma psicóloga, brasileira, com fluência na Língua Inglesa e conhecedora da finalidade da tradução. Nesta fase da validação, foi composto um comitê de três Juízes Especialistas em dependência química. $O$ trabalho destes profissionais consistia em comparar as versões do instrumento, verificando se os itens das duas versões do questionário referiam-se ou não ao tema "craving", bem como opinar a respeito de quais itens das duas versões traduzidas para a Língua Portuguesa eram mais adequadas à finalidade do instrumento. Os resultados devolvidos pelos membros do comitê foram levantados, sendo produzida a versão final do ACQ-SFR em Português.

6. Estudo Piloto: a versão final em Português foi aplicada em 20 sujeitos com as características da amostra pesquisada. O objetivo desta aplicação foi verificar, do ponto de vista destes pacientes, se o ACQ-SFR estava adequado tanto gramatical, quanto funcionalmente. No Anexo A, se encontra a versão final do ACQ-SFR em Português.

\section{Procedimentos}

\section{Aspectos éticos}

O início do processo de validação do ACQ-SFR ${ }^{12,15}$ somente ocorreu a partir do envio da autorização do seu autor para esta pesquisa, via correio eletrônico e após o projeto de pesquisa ser aprovado pelo Comitê de Ética em pesquisa do Hospital Psiquiátrico São Pedro (Resolução 13014). Antes de cada indivíduo aceitar fazer parte da pesquisa, mesmo durante 0 Estudo Piloto, foi explicada a finalidade da mesma, sendo fornecido um Termo de Consentimento Livre e Esclarecido, cuja assinatura era uma pré-condição para que o sujeito fosse incluído na amostra.

\section{Coleta de dados}

Cada participante que preenchesse os critérios de inclusão era encaminhado, individualmente, a uma sala onde era feita uma entrevista de avaliação. Foram então coletados os Dados sócio-demográficos e referentes ao padrão de consumo de substâncias psicoativas, o Mini-Mental, e analisado o diagnóstico pela CID-10. Após, foram apresentadas a todos duas fotos nas quais havia bebida alcoólica. Posteriormente, foram aplicados os instrumentos na seguinte ordem: ACQ-SFR, EAV, BAI e BDI.

\section{Análise de dados}

As informações coletadas nesta pesquisa foram organizadas e analisadas pelo software "Statistical Package for the Social Sciences"(SPSS) ${ }^{24}$ versão 18.0. A análise exploratória dos dados constou de testes estatísticos de frequências. A confiabilidade da escala foi avaliada através do teste de consistência interna alfa de Cronbach e o método split-half. Foi utilizado o teste Kolmogorov-Smirnov para avaliar se havia uma distribuição normal das variáveis, o que foi comprovado para todas elas, levando à utilização de testes Paramétricos. O Kaiser-Meyer-Olkin (KMO) e o teste de Bartlett foram utilizados com o intuito de comprovar a adequação dos dados do ACQ-SFR para a análise fatorial, permitindo que fosse utilizado o teste ANOVA. Os fatores foram avaliados a partir da utilização da rotação Promax. O critério utilizado para a colocação dos itens nos fatores foi o descrito por Tiffany e Drobes ${ }^{25}$, os quais consideraram, como pertencentes a um determinado fator, itens com carga fatorial igual ou superior a 0,40 , cuja carga no outro fator fosse menor do que 0,25 , devendo a diferença entre ambas ser de, no mínimo, 0,20. A validade discriminante foi avaliada pela comparação do escore total do ACQ-SFR, do escore de seus fatores e da pontuação na Escala Analógico-Visual em dois grupos divididos pela quantidade de álcool consumida por semana: até 144 Uis e 145 Uis ou mais. Essa divisão foi realizada a partir do cálculo do ponto médio da quantidade de álcool consumida. O Coeficiente de Correlação Linear de Pearson foi utilizado na validade convergente e de critério, bem como no estudo dos fatores associados ao craving e o Teste t de Student para amostras independentes, utilizado na validade discriminante e na análise de associações com o craving. O nível de significância utilizado como parâmetro foi o de $5 \%$.

\section{RESULTADOS}

Os participantes da amostra $(n=121)$ com Transtorno devido ao uso de álcool tinham, em média, 44, 72 anos, com Desvio-Padrão (DP) de $10,41$ e 7,69 anos de estudo ( $D P=2,60)$. Com relação ao estado civil: $41,3 \%$ eram solteiros, $31,4 \%$ eram casados ou tinham companheira, $22,3 \%$ eram separados ou divorciados e $5 \%$ viúvos. Eles iniciaram o uso de álcool, em média, com 15,41 anos (DP=4,52), consumiam 188,4 UI de álcool por semana 
( $D P=162,82)$ e usaram álcool pela última vez há cerca de 18,69 dias ( $D P=13,93)$. As médias obtidas no $B D I$ e BAI foram, respectivamente, 18,79 $(\mathrm{DP}=12,40)$ e $11,70(\mathrm{DP}=12,09)$ pontos. Na Tabela 1 podem ser observados os demais dados de caracterização da amostra referentes ao uso de outras substâncias.

\section{Confiabilidade}

O alfa de Cronbach total do ACQ-SFR foi de 0,91 (12 itens), do Fator 1 foi 0,92 (8 itens) e do Fator 2 foi 0,70 ( 3 itens). Com o método sses-half foi obtido o coeficiente 0,91 , sendo que a parte 1 ( 6 questões) teve um alfa de 0,84 e a parte 2 ( 6 questões), alfa de 0,83 ; a correlação entre as duas partes foi de 0,84 .

\section{Análise Fatorial}

Os resultados do Kaiser-Meyer-Olkin (KMO) e do teste de Bartlett foram, respectivamente, 0,885 e $p<0,001$ e, na Tabela 2, estão distribuídas as questões nos fatores da escala.

Os fatores 1 e 2 do ACQ-SFR apresentaram autovalor de 6,14 e 1,29 e variância de 51,18\% e $10,72 \%$, respectivamente, sendo o total da variância equivalente a $61,9 \%$.

\section{Validade Convergente e de Critério}

A validade convergente e de critério do ACQ-SFR foi mensurada, respectivamente, por meio da análise das correlações do ACQ-SFR entre seus fatores, e de seus fatores com os escores da Escala Analógico-Visual, o que pode ser observado na Tabela 3.

Tabela 1: Caracterização da amostra quanto ao uso de substâncias psicoativas $(n=121)$.

\begin{tabular}{lcc}
\hline \multicolumn{1}{c}{ Variáveis } & $\mathbf{n}$ & $\%$ \\
\hline $\begin{array}{l}\text { Transtorno por uso de álcool sem uso } \\
\text { de outras substâncias * }\end{array}$ & 78 & 64,5 \\
$\begin{array}{l}\text { Transtorno por uso de álcool com uso } \\
\text { de outras substâncias }\end{array}$ & 43 & 35 \\
Transtorno por uso de tabaco & 87 & 71,9 \\
Transtorno por uso de tabaco em & 4 & 3,3 \\
remissão & & \\
Transtorno por uso de maconha & 19 & 15,7 \\
Transtorno por uso de maconha em & 12 & 9,9 \\
remissão & & \\
Transtorno por uso de cocaína (crack) & 27 & 22,3 \\
Transtorno por uso de cocaína (crack) & 2 & 1,7 \\
em remissão & & \\
Transtorno por uso de cocaína & 22 & 18,2 \\
(inalada) & & \\
Transtorno por uso de cocaína & 13 & 10,7 \\
(inalada) em remissão & & \\
\hline *Salvo tabaco e cafeína. & &
\end{tabular}

\section{Validade Discriminante}

Os resultados da validade discriminante podem ser analisados na Tabela 4.

\section{Craving e Fatores Associados}

$O$ estudo correlacional entre o total de pontos do ACQ-SFR, o Fator 1 e o Fator 2 e as variáveis: idade, anos de estudo, último uso de álcool, idade de início do uso do álcool, quantidade de álcool consumida por semana em Uis, total de pontos do BDI e do BAI está apresentado na Tabela 5.

\section{DISCUSSÃO}

A versão brasileira do ACQ-SFR apresentou excelente consistência interna tanto na escala total com 12 itens como nos fatores 1 e $2^{25}$, confirmando que seus itens avaliam, de modo consistente, o constructo craving. Também foi possível comprovar a sua sensibilidade para medir as alterações no craving associado ao consumo de diferentes quantidades de bebidas alcoólicas, tanto pela pontuação total da escala, quanto pelo Fator 1, não sendo, porém, sensível no caso do Fator 2. Esse achado pode ser decorrente de que esse fator mede o Propósito de beber associado ao prazer, o que pode estar

Tabela 2: Análise Fatorial das questões do ACQ-SFR - Versão Brasileira $(n=121)$.

\begin{tabular}{lcc}
\hline \multicolumn{1}{c}{ ACQ-SFR } & Fator 1 & Fator 2 \\
\hline Questão 1 & $0,719^{*}$ & 0,159 \\
Questão 2 & $0,731^{*}$ & 0,032 \\
Questão 3 & $-0,017$ & $0,860^{*}$ \\
Questão 4 & 0,452 & 0,312 \\
Questão 5 & $0,773^{*}$ & 0,028 \\
Questão 6 & $0,848^{*}$ & $-0,075$ \\
Questão 7 & $0,911^{*}$ & $-0,095$ \\
Questão 8 & $-0,092$ & $0,951^{*}$ \\
Questão 9 & $0,758^{*}$ & 0,002 \\
Questão 10 & $0,771^{*}$ & 0,013 \\
Questão 11 & 0,146 & $0,506^{*}$ \\
Questão 12 & $0,811^{*}$ & $-0,009$ \\
\hline *Participa da composição do Fator.
\end{tabular}

Tabela 3: Correlações de Pearson do ACQ-SFR- Versão Brasileira, fatores 1 e 2 e Escala Analógico-Visual (EAV).

\begin{tabular}{lcccc}
\hline Amostra=121 & $\begin{array}{c}\text { ACQSFR } \\
\text { Total }\end{array}$ & Fator 1 & Fator 2 & EAV \\
\hline ACQ-SFR Total & 1 & $0,967^{*}$ & $0,702^{*}$ & $0,658^{*}$ \\
Fator 1 & $0,967^{*}$ & 1 & $0,521^{*}$ & $0,662^{*}$ \\
Fator 2 & $0,702^{*}$ & $0,521^{*}$ & 1 & $0,429^{*}$ \\
EAV & $0,658^{*}$ & $0,662^{*}$ & $0,429^{*}$ & 1 \\
\hline${ }^{*}<<0,01$ & & & &
\end{tabular}

${ }^{*} p<0,01$ 
Tabela 4: Comparação das médias (desvios-padrão) do craving entre grupos de acordo com a quantidade de álcool consumida por semana.

\begin{tabular}{lcccc}
\hline \multicolumn{1}{c}{ Variáveis } & Até 144 Uis & 145 Uis ou mais & T & p \\
\hline ACQ-SFR Total & $24,54(17,27)$ & $33,43(20,14)$ & $-2,590$ & $0,011^{*}$ \\
Fator 1 & $15,87(12,18)$ & $23,62(15,88)$ & $-3,011$ & $0,003^{*}$ \\
Fator 2 & $6,22(4,94)$ & $6,90(4,79)$ & $-0,767$ & 0,445 \\
EAV & $1,82(2,84)$ & $3,38(3,78)$ & $-2,568$ & $0,011^{*}$ \\
\hline
\end{tabular}

${ }^{*} \mathrm{p}<0,05$.

Tabela 5: Correlações de Pearson entre ACQ-SFR, Fator 1 e 2 com demais variáveis pesquisadas.

\begin{tabular}{lccc}
\hline \multicolumn{1}{c}{ Variáveis } & $\begin{array}{c}\text { ACQ-SFR } \\
\text { Total }\end{array}$ & Fator 1 & Fator 2 \\
\hline Idade & $-0,090$ & $-0,081$ & $-0,070$ \\
Anos de estudo & $-0,059$ & $-0,068$ & $-0,002$ \\
Último uso de álcool & 0,117 & 0,063 & $0,248^{* *}$ \\
Idade de início do &,$- 0235^{* *}$ & $-0,180^{*}$ & $-0,330^{* *}$ \\
uso do álcool & & & \\
Quantidade de & 0,107 & 0,172 & 0,074 \\
álcool consumida & & & \\
em Ul & & & \\
BDI total de pontos & $0,514^{* *}$ & $0,522^{* *}$ & $0,339^{* *}$ \\
BAI total de pontos & $0,514^{* *}$ & $0,514^{* *}$ & $0,387^{* *}$ \\
\hline${ }^{*} \mathrm{p}<0,05 ;{ }^{* *} \mathrm{p}<0,01$. & & &
\end{tabular}

diminuído na internação, onde é impossível, ao indivíduo, consumir o álcool.

$\mathrm{O}$ resultado quanto à divisão das questões nos dois fatores encontrados na versão brasileira foi diferente, tanto da validação realizada por Singleton et al. ${ }^{12,13}$, que encontraram quatro fatores, quanto da validação espanhola ${ }^{14}$, que encontraram três. O único ponto em comum foi que o Fator 2 da validação brasileira correspondeu às mesmas questões que compuseram o Fator 3 da validação original que se refere a "Propósito, impulsos e desejos, combinados com intenção e planejamento para beber"12,13. Na distribuição fatorial da validação americana, todas as 12 questões compuseram os quatro fatores ${ }^{12,13}$, enquanto, na brasileira, a questão 4 não teve sua carga fatorial, de acordo com os critérios estabelecidos ${ }^{25}$, participando de um único fator, o que levou à sua não-inclusão na soma dos pontos de cada um dos dois fatores, apenas no somatório total.

Os fatores 1 e 2, apesar de distintos, estão moderadamente correlacionados, porém essa correlação é menor do que 0,80 , confirmando que estes fatores não mensuram constructos idênticos ${ }^{26}$. Foi encontrada correlação de muito alta intensidade entre o Total de pontos do ACQ-SFR e o Fator 1, demonstrando haver redundância entre estas duas medidas e correlação alta com o Fator 2, mas, neste caso, os dois avaliam aspectos distintos. O Total de pontos também teve correlação alta com a EAV, sendo esta última correlacionada, com intensidade alta, com o Fator 1 e moderada, com o Fator 2. A associação entre o ACQ-SFR e a EAV já tinha sido pesquisada por Potgieter, Deckers e Geerlings ${ }^{15}$ que verificaram que a primeira escala avaliava os aspectos desejo (intensidade, distúrbios afetivos e estímulos) e pensamentos (sobre o controle do uso do álcool e expectativas positivas), enquanto que a segunda, mensurava apenas o desejo. As correlações de moderada a altas entre o Total de pontos do ACQ-SFR, seus dois fatores e a EAV realizadas na validação convergente demonstra que a versão brasileira da escala validada é competente na avaliação do craving.

O Total de pontos do ACQ-SFR e os Fatores 1 e 2 foram correlacionados negativamente, com intensidades baixas com a idade de início do uso do álcool e positivamente, com intensidades de baixas a moderadas, com sintomas de depressão (BDI) e de ansiedade (BAI). A correlação entre craving e sintomas de depressão e ansiedade já foi verificado em outros estudos ${ }^{27,28}$. Apesar das médias de pontos do BAI e BDI não terem sido altas, a presença dessa correlação indica a importância da avaliação de comorbidades psiquiátricas em alcoolistas, bem como do craving como forma de aliviar o afeto negativo, o que já havia sido descrito por outros autores ${ }^{12-14,22,25}$.

Pesquisadores do craving costumam defender a ideia de que as escalas unidimensionais - como as EAV - não são efetivas na avaliação deste constructo, devendo optar-se por escalas multidimensionais como o ACQ-SFR ${ }^{12,13,22,25}$. Deve-se observar que a validação brasileira foi realizada com sujeitos que eram, na sua totalidade, do sexo masculino e que estavam internados para desintoxicação, o que pode ter interferido nos seus resultados. Outra limitação foi o fato de não ser utilizado um grupo-controle para o qual fosse apresentado fotos com um estímulo neutro, o que sugere-se para novos estudos.

A construção e validação de escalas para avaliação do craving por substâncias psicoativas é 
algo importante para que este constructo possa ser melhor compreendido e manejado na prática clínica.

\section{CONCLUSÃO}

A versão brasileira do ACQ-SFR é uma escala que apresenta propriedades psicométricas satisfatórias, sendo efetiva para a avaliação do craving, de uma forma multidimensional, em indivíduos com Transtorno por uso do álcool. O fato dele ser uma escala breve, torna, a sua aplicação, mais fácil, rápida e barata, favorecendo, ao clínico, a identificação da necessidade da utilização de técnicas para o manejo do craving para a prevenção de recaídas.

\section{Agradecimentos}

Ao Dr. Edward Singleton (EUA), pela autorização dada para a validação do ACQ-SFR no Brasil e para a publicação da versão brasileira dessa escala.

\section{Conflitos de Interesse}

Os autores declaram não ter conflitos de interesse.

\section{REFERÊNCIAS}

1. Araujo RB, Oliveira MDS, Pedroso RS, Miguel AC, Castro MGT. Craving e dependência química: conceito, avaliação e tratamento. $J$ Bras Psiquiatr. 2008;57(1):57-63. http://dx.doi.org/10.1590/S004720852008000100011.

2. Sayette MA, Shiffman S, Tiffany ST, Niaura RS, Martin CS, Schadel WG. The measurement of drug craving. Addiction. 2000;95(8 Suppl 2):189210. http://dx.doi.org/10.1046/j.13600443.95.8s2.8.x. PMid:11002914.

3. Araujo, RB. Guia de terapias cognitivo-comportamentais para os transtornos do exagero: tratando pacientes da vida real. Novo Hamburgo: Editora Sinopsys; 2013

4. Organização Mundial de Saúde (OMS). Classificação de transtornos mentais e de comportamento da CID10: descrições clínicas e diretrizes diagnósticas. Porto Alegre: Artes Médicas; 1993.

5. American Psychiatric Association. DSM-5: manual diagnóstico e estatístico de transtornos mentais. 5th ed. Porto Alegre: Artmed; 2014.

6. Gass JC, Tiffany ST. Craving and tobacco use: development of the choice behavior under cued conditions (CBUCC) procedure. Psychol Addict Behav. 2017;31(3):276-83. http:// dx.doi.org/10.1037/adb0000259. PMid:28240934.

7. Beck AT, Wright FW, Newman CF, Liese B. Cognitive therapy of substance abuse. New York: Guilford Press; 1993.

8. Singleton EG, Tiffany ST, Henningfield JE. Development and validation of a new questionnaire to assess craving for alcohol. In: Harris LS, editor. Proceedings of the $56^{\text {th }}$ Annual Scientific Meeting - The College on Problems of Drug Dependence; 1994 June 18-23; Palm Beach, Florida. Rockville: National Institute on Drug Abuse; 1994. p. 289-289.

9. Singleton EG, Henningfield JE, Heishman TE, Douglas E, Tiffany ST. Multidimensional aspects of craving for alcohol. In: Harris LS, editor. Proceedings of the 57th Annual Scientific Meeting - The College on Problems of Drug Dependence; 1995 June 10-15; Rockville, Maryland. Rockville: National Institute on Drug Abuse; 1995.

10. Love A, James D, Willner $P$. A comparison of two alcohol craving questionnaires. Addiction. 1998;93(7):1091-102. http:// dx.doi.org/10.1046/j.13600443.1998.937109113.x. PMid:9744139.

11. Raabe A, Grüsser SM, Wessa M, Podschus J, Flor H. The assessment of craving: psychometric properties, factor structure and a revised version of the Alcohol Craving Questionnaire (ACQ). Addiction. 2005;100(2):22734. http://dx.doi.org/10.1111/j.13600443.2005.00960.x. PMid:15679752.

12. Singleton EG. Alcohol Craving Questionnaire, Short-Form (Revised) (ACQ-SF-R): background, scoring, and administration - Unpublished research, Behavioral Pharmacology Research Unit (BPRU). Baltimore: Department of Psychiatry and Behavioral Sciences, The Johns Hopkins University School of Medicine; 1997.
13. Singleton EG, Tiffany ST Henningfield JE. Manual Alcohol Craving Questionnaire (ACQNow): background, scoring, and administration - Unpublished manuscript. Baltimore: Division of Intramural Research; 1994. 28 p.

14. Gálvez BP, Maroto JJ, Fernández LG, Manzanaro MPV, Ivorra NC. Validación de tres instrumentos de evaluación del craving al alcohol ver ver muestra española. Health and Addictions. 2016;16(2): 73-9.

15. Potgieter AS, Deckers F, Geerlings $P$. Craving and relapse measurement in alcoholism. Alcohol Alcohol. 1999;34(2):254-60. http://dx.doi. org/10.1093/alcalc/34.2.254. PMid:10344785.

16. Hair JF JR, Anderson RE, Tatham $\mathrm{RL}$, Black WC. Análisis multivariante. Madrid: Prentice Hall Iberia; 1999.

17. Folstein MF, Folstein SE, McHugh PR. Mini-mental state: a practical method for grading the cognitive state of patient for the clinician. $J$ Psychiatr Res. 1975;12(3):189-98. http://dx.doi. org/10.1016/0022-3956(75)90026-6. PMid:1202204.

18. Oliveira M. Eficácia da intervenção motivacional em dependentes do álcool [tese]. São Paulo: Universidade Federal de São Paulo; 2000.

19. Beck AT, Steer RA. Beck anxiety inventory - manual. San Antonio: Psychological Corporation; 1993.

20. Cunha VER. Manual da versão em português das Escalas Beck. São Paulo: Casa do Psicólogo; 2001. 
21. Beck AT, Steer RA. Beck depression inventory - manual. San Antonio: Psychological Corporation; 1993.

22. Araujo RB, Oliveira MS, Moraes JFD, Pedroso RS, Port F, Castro MGT. Validação da versão brasileira do Questionnaire of Smoking Urges- Brief. Ver Psiquiatr Clín. 2007;34(4):166-75.

23. Pedroso RS, Oliveira MS, Moraes JFD. Tradução, adaptação e validação da versão brasileira da escala Marijuana Expectancy Questionnaire. Cad Saude Publica. 2007;23(1):6373. http://dx.doi.org/10.1590/ S0102-311X2007000100008. PMid:17187105.
24. Rowland D, Arkkelin D, Crisler L. Computer-based data analysis: using SPSSxi in the Social and Behavioral Sciences. Chicago: Nelson-Hall; 1991.

25. Tiffany ST, Drobes DJ. The development and initial validation of a questionnaire on smoking urges. $\mathrm{Br}$ J Addict. 1991;86(11):1467-76. http:// dx.doi.org/10.1111/j.1360-0443.1991. tb01732.x. PMid:1777741.

26. Ferrans CE, Powers MJ. Psychometric assessment of the Quality of Life Index. Res Nurs Health. 1992;15(1):29-38. http:// dx.doi.org/10.1002/nur.4770150106. PMid:1579648.
27. Fox HC, Bergquist LL, Hong K, Sinha R. Stress-induced and alcohol cueinduced craving in recently abstinent alcohol-dependent individuals. Alcohol Clin Exp Res. 2007;31(3):395-403. http://dx.doi.org/10.1111/j.15300277.2006.00320.x. PMid:17295723.

28. Willner P, Field M, Pitts K, Reeve G. Mood, cue and gender influences on motivation, craving and liking for alcohol in recreational drinkers. Behav Pharmacol. 1998;9(7):631-42. http://dx.doi.org/10.1097/00008877199811000-00018. PMid:9862088.

Recebido: 2 out, 2018 Aceito: 27 jan, 2019 
Anexo A: Versão brasileira do Alcohol Craving Questionnaire - Short Form - Revised - Versão Brasileira (ACQ-SF-R)

ALCOHOL CRAVING QUESTIONNAIRE-SHORT FORM-REVISED - VERSÃO BRASILEIRA(ACQ-SF-R) Por gentileza, indique o quanto você concorda ou discorda de cada uma das frases abaixo fazendo um "x" (assim: X_) em cada número, ao longo de cada linha entre DISCORDO TOTALMENTE e CONCORDO TOTALMENTE. Quanto mais perto de cada uma das pontas você marcar, isso indicará o quanto você concorda ou discorda. Estamos interessados em saber como você está pensando ou sentindo agora, enquanto você preenche este questionário. Por favor, preencha todos os itens.

1. Se eu tivesse alguma bebida alcoólica, eu provavelmente a beberia.

DISCORDO TOTALMENTE $1: 2: 3: 4: 5: 6: 7$ CONCORDO TOTALMENTE

2. Eu sinto falta de beber.

DISCORDO TOTALMENTE $1: 2: 3: 4: 5: 6: 7$ CONCORDO TOTALMENTE

3. Eu não estou planejando, de forma alguma, tomar uma bebida.

DISCORDO TOTALMENTE $1: 2: 3: 4: 5: 6: 7$ CONCORDO TOTALMENTE

4. Eu não conseguiria deixar de beber se eu tivesse alguma bebida alcoólica aqui. DISCORDO TOTALMENTE $1: 2: 3: 4: 5: 6: 7$ CONCORDO TOTALMENTE

5. Eu quero tanto beber que posso quase sentir o gosto.

DISCORDO TOTALMENTE $1: 2: 3: 4: 5: 6: 7$ CONCORDO TOTALMENTE

6. Eu me sentiria menos irritado(a) se eu pudesse tomar uma bebida agora.

DISCORDO TOTALMENTE $1: 2: 3: 4: 5: 6: 7$ CONCORDO TOTALMENTE

7. Se eu bebesse agora, eu me sentiria menos tenso.

DISCORDO TOTALMENTE $1: 2: 3: 4: 5: 6: 7$ CONCORDO TOTALMENTE

8. Beber não seria muito gratificante.

DISCORDO TOTALMENTE $1: 2: 3: 4: 5: 6: 7$ CONCORDO TOTALMENTE

9. Eu me sentiria menos agitado se eu bebesse álcool.

DISCORDO TOTALMENTE $1: 2: 3: 4: 5: 6: 7$ CONCORDO TOTALMENTE

10. Se eu estivesse bebendo, eu me sentiria menos nervoso.

DISCORDO TOTALMENTE $1: 2: 3: 4: 5: 6: 7$ CONCORDO TOTALMENTE

11. Seria fácil deixar passar a oportunidade de beber

DISCORDO TOTALMENTE $1: 2: 3: 4: 5: 6: 7$ CONCORDO TOTALMENTE

12. Uma bebida me faria sentir melhor.

DISCORDO TOTALMENTE $1: 2: 3: 4: 5: 6: 7$ CONCORDO TOTALMENTE 